\title{
Development and validation of scoring systems for the risk of cerebral infarction
}

Toshiyuki Hijikata', Tsubasa Ito', Megumi Nishizaka', Takahiro Mihara²

${ }^{1}$ Department of emergency medicine and intensive care, Itabashi chuo medical center, Tokyo, Japan

2Department of anesthesiology, Yokohama City University of Medicine, Yokohama, Japan

\section{Background and Goal of this study}

- Patients suspected to have cerebral infarction $(\mathrm{Cl})$ often present at the emergency department.

- $\mathrm{MRI}$ is one of tool to make a diagnosis of $\mathrm{Cl}$. However it is high cost of medical care.

- Reliable prediction methods reduce extra imaging test and make clinicians to decide early treatment

\section{The goal of this study was to develop and validate}

\section{a risk score for predicting $\mathrm{Cl}$}

\section{Methods and Results}

【Data】

Data used in this study were from 1,553 patients suspected to have $\mathrm{Cl}$ and were examined using $\mathrm{CT}$ and MRI the same day.

【Outcome】

Patient was $\mathrm{Cl}$ or not. ( $\mathrm{Cl}$ was diagnosed by a radiologist or neurologist using $\mathrm{MRI}$ imaging )

【predictors】

56 candidate predictors was selected (e.g.: demographic data, PMH, medications, physical findings, blood exam, imaging findings )

Parameter were missing for more than $20 \%$ were excluded

All parameters were evaluated for multicollinearity, correlation was detected $(>0.7)$, only a single parameter was included.

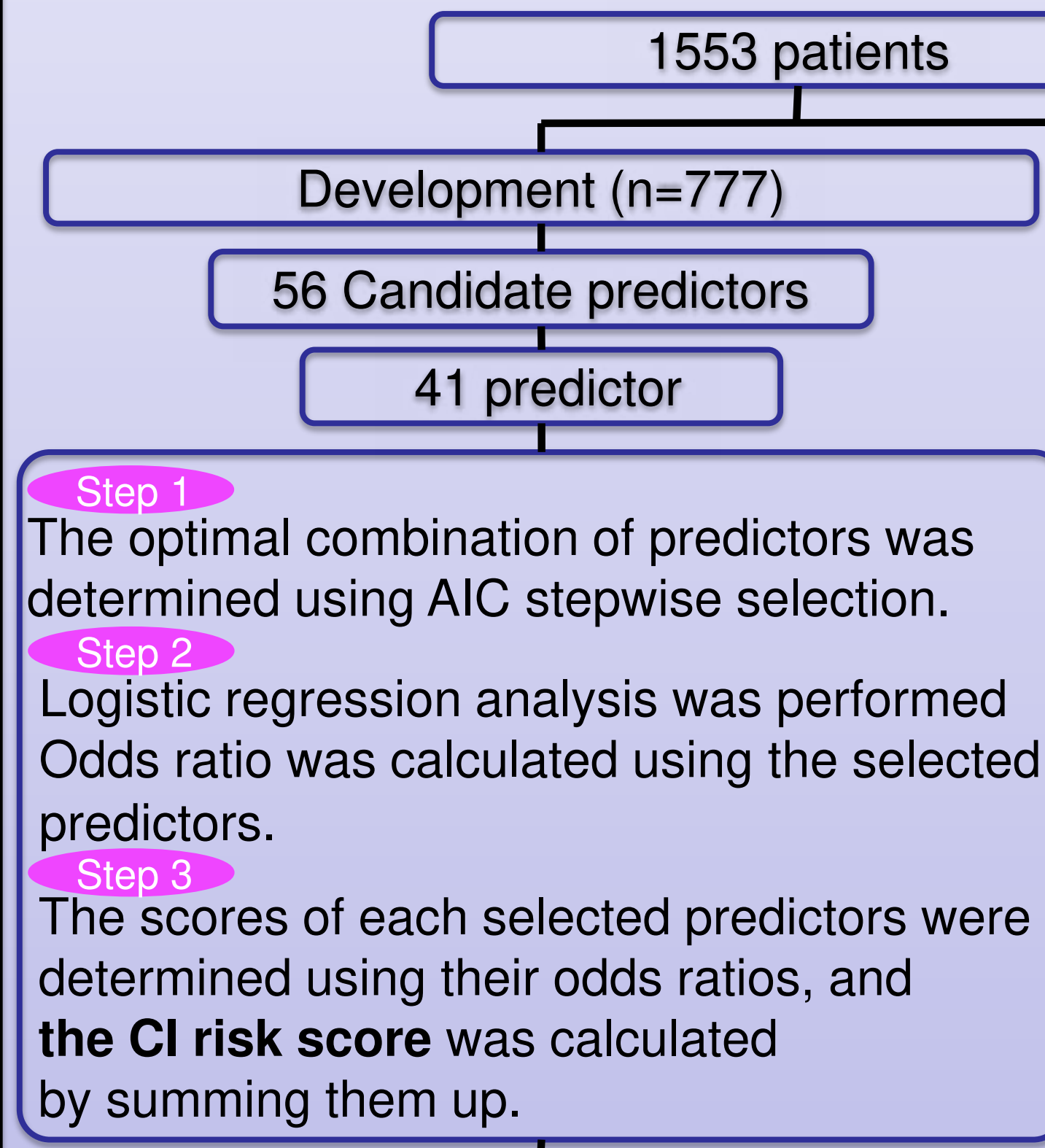

The $\mathrm{Cl}$ risk score of 12 selected predictors (Table1)

Validation $(\mathrm{n}=776)$

Step 4

The predictive ability of the $\mathrm{Cl}$ risk score was examined by generating an ROC curve $(\Rightarrow)$ and gray zone approach.

AIC Akaike'----------AIC(Akaike's information criteria) is an index for choosing the most optimal statistical model (i.e., the most optimal combination of predictors)

The gray zone was determined as the range between the two points where sensitivity and specificity became $90 \%$ each.

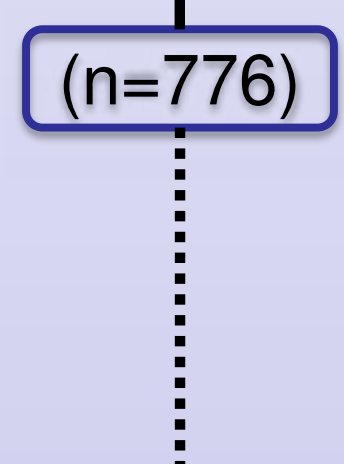

Table1: Cl risk score

\begin{tabular}{|lc|}
\hline Values & score \\
\hline Onset & \\
$\quad<24 \mathrm{~h}$ & 2 \\
Vital sign and physical findings & \\
$\quad$ Mean blood presser (mmHg) & 0.05 \\
$\quad$ Barre's sign & 2 \\
$\quad$ Paralysis of limbs & 2 \\
$\quad$ Visual defect & 9 \\
$\quad$ Headache & -2 \\
Social and Past history & \\
$\quad$ Smoking & 1 \\
$\quad$ Epilepsy & -10 \\
$\quad$ Stroke & -1 \\
Blood exam & \\
$\quad$ APTT & $x-0.1$ \\
$\quad$ PT-INR & x 3 \\
Imaging findings & \\
$\quad$ Acute low density area of CT & 8 \\
\hline Total & The Cl risk score \\
\hline
\end{tabular}

I

\section{Conclusion}

We established and validated the $\mathrm{Cl}$ risk score with relatively high accuracy. This risk score is useful for not only predicting $\mathrm{Cl}$ but also deciding on early treatment in clinical settings. 